\title{
COMPORTEMENT ALIMENTAIRE DU LAPIN DE GARENNE EN CAPTIVITÉ
}

\author{
M. PRUD'HON et J. GOUSSOPOLLOS \\ Station de Physiologie animale, E. N. S. A., \\ Centre de Recherches de Montpellier, I. N.R. A., \\ 9. Place Viala, \\ 34060 Montpellier Cedex \\ RÉ,SUMÉ:
}

Les caractéristiques du comportement alimentaire de six lapins de garenne élevés en cage ct recevant de l'eau et de l'aliment granulé ont été enregistrées. Les fréquences des prises, les quantités absorbées et la répartition de la consommation au cours du nycthémère ont été analysées. Comparé au Lapin domestique, le Lapin de garenne se caractérise par une consommation quasi nulle entre l'heure qui précède l'allumage et 2 à 3 heures avant l'extinction des fcux. Un petit repas solide est parfois réalisé en milieu de période claire.

Chez les garennes étudiés ici, le rapport eau/aliment $(\mathrm{I}, 20$ à 1,30$)$ est sensiblement plus faible que chez des lapins domestiques de même âge placés dans les mêmes conditions.

\section{INTRODUC'TION}

S'il existe de nombreuses clonnées d'observation sur le comportement du Lapin de garenne en liberté, notamment l'aspect crépusculaire et nocturne de sa consommation d'aliments, on a peu de renseignements précis sur les caractéristiques de son comportement alimentaire lorsqu'il est élevé en captivité.

Il est intéressant en particulier d'étudier la répartition nycthémérale de ses prises d'aliment; on sait, en effet, que le Lapin domestique a un comportement nocturne prédominant (Cheviliard, Gasiter et Mayer, i939: Prud'hon et al., i972; Sanderson et Vaxderweele, 1975) mais avec des variations importantes entre individus et même, intra-individu, en fonction de facteurs non génétiques tels que l'âge (PrUd'HON et al., 1975), la température ambiante (PRUD'HON, I976), etc. Il est possible que l'aptitude à ingérer des aliments tout au long du nycthémère soit un caractère sous contrôle génétique. La comparaison entre les comportements des lapins de garenne et clomestiques, placés dans les mêmes conditions d'élevage, devrait permettre de mesurer le chemin parcouru en ce domaine lors de la domestication.

\section{MATÉRIEL ET MÉTHODES}

Six lapins de garenne, quatre mâles et deux femelles, appartenant à deux portées différentes, élevés en cages individuelles sur grillage depuis le sevrage, ont été placés à l'âge de 9 mois environ, dans le dispositif d'enregistrement graphique des consommations décrit précédemment (PrU- 
D'Hox et al., 1972). L'une des portées avait été recueillie directement au nid lors de travaux agricoles, l'autre était issue de l'accouplement de deux lapins de la première portéo (unique naissance en captivité que nous ayons obtenue).

Les lapins étaient éclairés de 7 heures à 2 I heures pour ne pas modifier le régime lumineux reçu précédemment; ils avaient la possibilité de se réfugier clans un abri obscur placé à l’intéric'ur cle leurs cages.

La température était maintenue comstante à $20 \therefore$ IoC.

Les lapins recevaient à volonté de l'eau, dans un abreuvoir a niveau constant, de l'aliment granulé closant au minimum i6 p. Ioo de protéines brutes et $\mathrm{I}+\mathrm{p}, 100$ (le cellulsse.

L'enregistrement a été poursuivi sans interruption pendant trois semaines (première portéc) et quatre semaines (deuxième portée) ; la table d'enregistrement était située dans la pièce contiguë à la salle de mesure ; les lapins n'étaient visités qu'occasionnellement, une fois par semaine environ, jamais à heure fixe ct pendant un bref laps de temps.

Aucune pesée des animaux n'a été réalisée a cours de cette étude en raison cles faibles coffectifs disponibles, des risques d'accidents en cours de manipulation et des perturbations que peuvent occasionner cette opération chez un tel type d'animal. Les lapins ont été abattus is jours cnviron après la fin des enregistrements. 1ls pesaient respectivement : première portée: i ouo, I loo et I I $60 \mathrm{~g}$; deuxième portée: I 095 , I 120 et $870 \mathrm{~g}$.

\section{RÉSULTATS E'T DISCUSSION}

\section{TABIEAU I}

Principales caractéristiques du comportement alimentaive du lapin de gareme

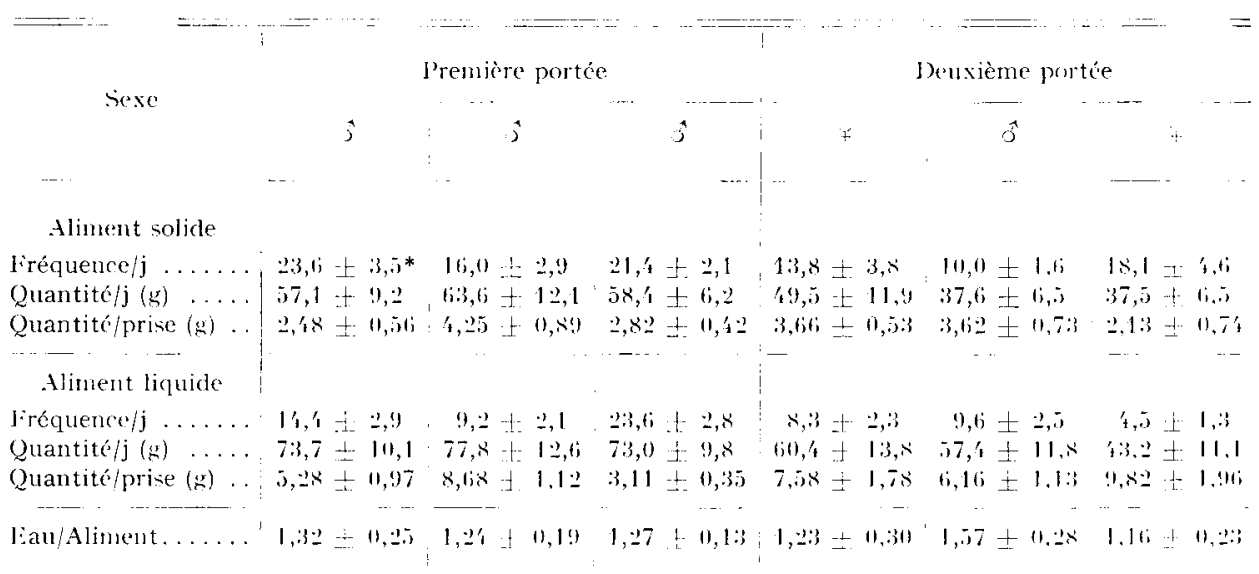

* Moyenue $\doteq$ éartype.

Comme le lapin domestique, he Iapin de garenne effectue de multiples prises d'aliment ( 10 à 20 prises solicles par jour, 5 à 20 prises liquides) la fréquence dess prises étant très variable d'un individu à l'autre, même au sein d'une portée, et d'un jour à l'autr’s (tabl. 1). la a prise: moyenne varie de 2 à $+\mathrm{g}$ pour le granulé, de 3 à ro g pour l'eau.

Quantitativement, les lapins de garenne étucliés ont consommé environ fo à tor y par jour et bu 45 à $80 \mathrm{~g}$ d'eau, les lapins de la première portée mangeant et buvant signilicativement plus que les lapins de la seconde portée mais étant également plus développés.

Lc rapport cau/aliment, proche de r, 20 à r, 30 était très sensiblement plus faible que (wui que l'on enregistre, dans des conditions comparables chez les lapins clomestiulues. 
Par rapport au Lapin domestique, la principale différence de comportement réside dans la répartition des repas au cours du nycthémère. La figure $x$ représente le pourcentage de la consommation quotidienne consommé au cours de chacune des heures du nycthémère. Nous avons retenu la valeur moyenne des six sujets étudiés chacune des courbes inclividuclles étant pratiquement superposable.
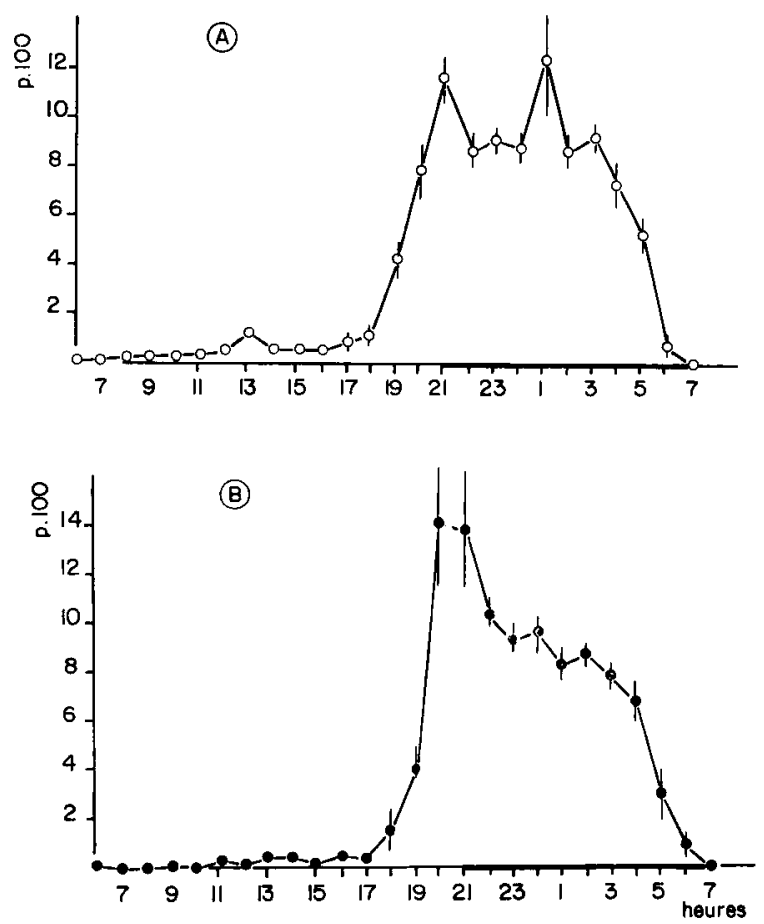

Fig. I. - ... Répartition au cours du nycthémère de la consommation relative de granule (A) et d'eau (13) de Lapins de garenne

(La période d'obscurité va de 2 I heures à 7 heures

La courbe représente la moyenne de six données individuelles assortie des écarts-typej

Le Lapin de garenne s'alimente presque exclusivement pendant les deux à trois heures qui précèdent l'extinction des lumières et pendant la plus grande partic le la nuit à l'exception de l'heure qui précède la réapparition de la lumière.

La consommation d'eau présente un pic particulièrement marqué avant ct après l'extinction des leux, et non en fin de nuit comme c'était le cas, en fin de croissance, chez les lapins domestiques (Prud'hor et al., 1975).

En ce qui concerne l'aliment granulé, il existe doux pics, l'un dans l'hcure qui suit l'extinction, l'autre en milieu de période obscure. Il existe égalemant chez tous les sujets étudiés, une consommation occasionnelle d'aliments solides, de faible amplitude, vers le milieu de la période claire, alors que lá prise de boisson à cette période est beaucoup plus rare.

En conclusion, le déroulement général des séquences cl'alimentation du Lapin cle garenne et du Lapin domestique sont très semblables mais les séquences sont presque exclusivement nocturnes chez le Garenne alors qu'clles sont réparties sur une fraction importante du ṇ̣cthémère chez le Lapin domestique. 
SUMMARY

FEEDING BEHAVIOUR OF WILD RABBITS IN CAPTIVITY

Feeding behaviour characteristics of six wild rabbits raised in cages and receiving water and pellets were recorded. Eating frequencies, amounts absorbed and distribution of consumptions during the circadian cycle were analysed. In comparison with domestic Rabbits, the wild Rabbits almost did not eat during the period ranging between one hour before switching on of the light and 2 to 3 hours before switching off of the latter. They were sometimes eating a small solid meal in the middle of the light period.

In the wild rabbits studied here, the water to feed ratio ( $\mathbf{r} 20$ to $\mathbf{r} .30$ ) was substantially lower than in domestic rabbits of the same age placed in the same conditions.

\section{RÉFÉRENCES BIBLIOGRAPHIQUES}

Cheviliard L., Gasier A., Mayer A., I939. Les modalités de l'ingestion chez les Iapins qui s'alimentent spontanément. Ann. Physiol. Physicochim. biol., 15, 27г-281.

Prud'hon M., Carles Y., Goussopoulos J., Koehl P. F., I972. Enregistrement graphique des consommations d'aliments solide et liquide du lapin domestique nourri ad libitum. Ann. Zootech., 21, 45 I-460.

Prud'hon M., Cherubin M., Golissopoulos J., Carles Y., i975. Évolution au cours de la croissance des caractéristiques de la consommation d'aliments solide et liquide du Lapin domestique nourri ad libitum. Ann. Zootech., 24, 289-298.

PRUd'hon M., r976. Comportement alimentaire du Lapin soumis aux températures de ro, 20 et $30^{\circ} \mathrm{C}$. Congrès int, cunicole. Dijon $3 \mathrm{I}$ mars-2 avril 1976.

Sanderson J. 1)., Vanderweele D. A., r975. Analysis of feeding patterns in normal and vagotonized rabbits. Physiol. Bchav., 15, 357-364. 